\begin{tabular}{|c|c|c|c|c|c|}
\hline 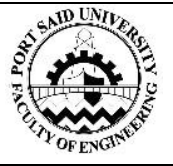 & \multicolumn{4}{|c|}{$\begin{array}{l}\text { PORT SAID ENGINEERING RESEARCH JOURNAL } \\
\text { Faculty of Engineering - Port Said University }\end{array}$} & 1 \\
\hline
\end{tabular}

\title{
A Novel Improvement in LEACH Protocol for Decreasing Energy Loss in Wireless Sensor Networks
}

\author{
Samar Awad Mohammed ${ }^{1}$, Khaled Abd Elsalam Aly², Atef Mohammed Ghuniem ${ }^{3}$
}

\begin{abstract}
One of the most important Internet of Things technologies is the Wireless Sensor Network (WSN), and it has recently gained a lot of interest because of its applications in monitoring, army, health, automation, agricultural, environment, and underwater. Two of the most important requirements for observing and detecting wireless topics within WSN are clarity of information disseminated and energy efficient. As a result, several forms of study are carried out. Many routing protocols that are based on topology with a hierarchical and clustering structure have been created to give a better result for deducing the network's energy exhaustion in WSN. However, these protocols do not consider all the necessary and important parameters for extending network life. In this suggested paper, we introduce two new methods to boost the WSN which raises energy-efficient and network lifetime. The simulated results of the proposed approaches are then compared to the prime LEACH Protocol with fixing parameters. When compared to the basic LEACH protocol in terms of energy-efficient and network lifetime, the proposed techniques outperformed it.
\end{abstract}

Keywords: LEACH, wireless sensor, Internet of Things

\section{INTRODUCTION}

WSN is now widely utilized in a variety of observation systems, including medical, military, agricultural, and commercial [1-2]. WSN is made up of many very small sensing nodes that are often powered by batteries. Several difficult functions are done by these tiny and inexpensive sensor nodes as collecting, processing, aggregating, and compressing the environmental data although the restriction of the energy of batteries. So, this battery limit may have an impact on energy-efficient [3-4]. Many research studies for this issue are performed to decrease the energy dissipation and prolong the network lifetime.

Routing protocols [5-6] that are dependent on the clustering approach are focused on several research studies. Routing protocols take over the clustering process in order to prevent network energy loss [7]. One of the energy-efficient routing algorithms is the Low Energy Adaptive Clustering Hierarchy (LEACH). Each cluster in the LEACH network has a Cluster Head $(\mathrm{CH})$ that connects with the Base Station (BS) and cluster member nodes, aggregating data from the nodes and transferring it to the (BS). [8]. A CH offers additional capabilities. We propose two unique energy-saving tweaks to the LEACH algorithm in this study. First one is Merged Transmitted Data LEACH (LEACH-MTD) by replacing the cluster head every round by checking if the remaining energy of $\mathrm{CH}$ still in the specified range, the $\mathrm{CH}$ in the previous round still the $\mathrm{CH}$ in the current round. Every round, $\mathrm{CH}$ will not communicate the data collected from its member

1 Electrical Department, Faculty of Engineering, suez canal University, Port-said, Egypt, email: samar.badr86@gmail.com, Corresponding author

2 Electrical Department, Faculty of Engineering, Suez canal University, Cairo, Egypt, email: khalid.aly14@gmail.com

Electrical Department, Faculty of Engineering, Suez canal University, Cairo, Egypt, email: atmohagh@gmail.com

Received: 12-06-2021, Accepted: 03-08-2021

DOI: 10.21608/PSERJ.2021.80413.1121 nodes to the base station. but it can hold it and merge it to the recently collected data in the next $m$ numbers of rounds according to the application designed for WSN. by this technique, we reduce energy consumption caused by $\mathrm{CH}$ transmission to $\mathrm{BS}$ and decrease overhead caused by $\mathrm{CH}$ advertisement. We notice that if $\mathrm{CH}$ has not processed the collected data in a correct way", the packet size of transmitted data from $\mathrm{CH}$ to the $\mathrm{BS}$ will be increased.

So, in the second enhancement LEACH via Schedule Off Technique With Merging (LEACH-SOTWM), we make member nodes divided into $\mathrm{m}$ groups only one group sends the sensing data to its $\mathrm{CH}$ in the existing round, and the other send in the next specified number of rounds, unlike the LEACH-MTD. We discovered that because the distance between member nodes in the same cluster is quite modest, there is no need for all of them to relay their sensing data to their $\mathrm{CH}$. Consequently, the sensed data from nodes in the same cluster are close in the same cluster. By that, we assure that the data packet size still fixed and there is no increase in energy consumption.

The following rules apply to this paper: The previous and related work is displayed in Section 2. The (LEACH) protocol is discussed in Section 3. The first updating technique is described in Section 4. The second update approach is discussed in Section 5. The experimental approach is shown in Section 6, and the results are examined in relation to the performance evaluation of our approaches. Finally, in Section 7, the conclusion is presented.

\section{RELATED WORK}

The wireless sensor area of research studies is rapidly grown, especially with many novel applications on the Internet of Things (IoT) domain. On another hand, there are many kinds of research are coming out with a set of 
techniques and protocols to develop and enhance This technology is attempting to cover the current requirements. The low battery is considered an obstacle to sensor Technologies. As a result, most of the following studies take these flaws into account and suggest approaches and algorithms to overcome them.

Sharma [9] described the development in the LEACH protocol in a system with heterogeneous status and contrasted the results with homogeneous network LEACH, which they used to simulate the protocol $100 *$ 100 meters zone. Sharma has discovered a boost in network longevity and performance due to the extra energy that 10 nodes have over the remaining 90 nodes.

Naveen [5] suggested fifteen distinct protocols have clustering wireless sensortrait, all of which he claimed were more energy-efficient and had a longer network lifetime. They contrast them to energy efficiency, load balancing, scalability, and stability, among other things. Prasad [11] proposed that a Cluster head picking algorithm to be considered as the principle for achieving better performance in clustering wireless sensor networks than others, as well as an improved energy-efficient leach protocol (IEELEACH) aimed at MANET with the goal of reducing energy losses and increasing network lifetime by selecting the $\mathrm{CH}$ that is closest to the BS and exhausting high residual energy. The increasing number of dead nodes is one of the reasons for the network's dereliction of duty.

Nandi [12] used a novel protocol for determining the ideal site for the BS, which addresses data transport issues, and compared the simulated results to the standard LEACH technique utilizing the TDMA approach. When the BS is positioned further away from the node, more energy is consumed at the node for data transmission from the node to the BS, resulting in a shorter network lifetime. Sharma [13] examined the throughput data and communication time of three distinct WSN protocols, including direct transmission protocol, $\mathrm{LEACH}$, and EEE LEACH. In comparison to other protocols, EEE LEACH has a faster data transmission time and a greater minimum throughput.

Sharma proposed the Distance Based Cluster Head (DBCH) algorithm in [14].

\section{LEACH}

Heinzelman et al. [15] proposed the LEACH process for WSNs, which is one of the main routing protocols that introduced the notion of clustering into the WSNs field, where the $\mathrm{CH}$ is chosen at random (1). Every round, each normal node gathers data from the environment and sends it to its $\mathrm{CH}$. The $\mathrm{CH}$ 's job was to receive all collected data from its member nodes in the same cluster, process it, compress it, and transfer it to the $\mathrm{BS}$ in a single transmission [14].

The LEACH protocol is divided into two steps: cluster forming step and steady-state step. In the first step, every sensor node adopts a value belongs to the interval $[0,1]$. If this is the case that value is lower than the threshold $T(n)$ giving in (1), this node becomes $\mathrm{CH}$ next round. Unlike that, it still a member node.

$$
T(\mathrm{n})=\left\{\begin{array}{cc}
\frac{\mathrm{p}}{1-\left(\mathrm{p} *\left(\mathrm{r} * \bmod \left(\frac{1}{\mathrm{p}}\right)\right)\right)} & \text { where } \mathrm{n} \in \mathrm{G} \\
0 & \text { other }
\end{array}\right.
$$

Here, $\mathrm{p}$ represents the likelihood of a node becoming a cluster head, $r$ represents the number of rounds, and $\mathrm{G}$ represents the set of nodes that have not been elected in the previous $1 / \mathrm{p}$ rounds. The $\mathrm{CH}$ creates TDMA slots for each node in a cluster since every member node is aware of the $\mathrm{CH}$ (to prevent interference). After that, the LEACH method moves on to the steady-state phase. All member node data packets are then delivered to the cluster head, who aggregates them and sends the resulting data to the BS. One of the LEACH algorithm's constraints is that the cluster head functions are larger than the member nodes, suggesting that the cluster head spends more energy than the other nodes. LEACH ignores the BS and cluster head position, instability, and energy dissipation in the case of cluster head death. The LEACH topological structure is shown in Figure 1.

The following kinds of attacks reduce the performance of LEACH by altering, dropping, spoofing, or replying to the packets attack The LEACH protocol.

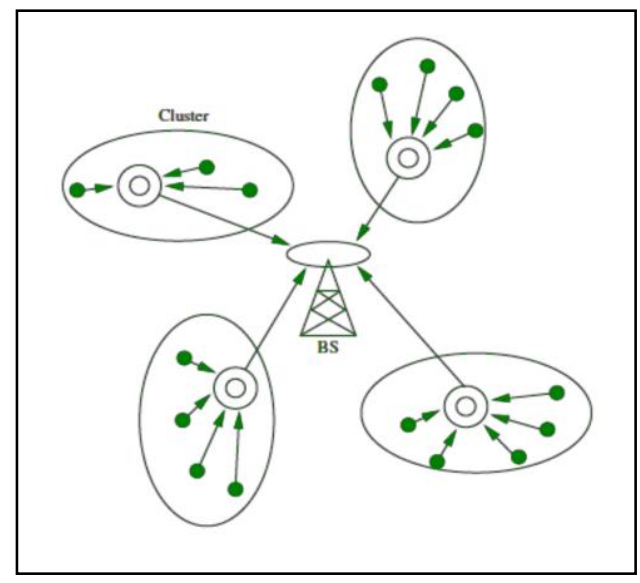

Figure 1. LEACH topology structure [15]

Generally, the energy is seen as a firmly connected operator to the network lifetime. In $\mathrm{LEACH}$, each $\mathrm{CH}$ connects to the $\mathrm{BS}$ in a single hop regardless of distance or available energy, which is not available to $\mathrm{CHs}$ at the network's rim. Distance plays a significant role on energy use.

The LEACH flowchart is declared in Figure 2. 


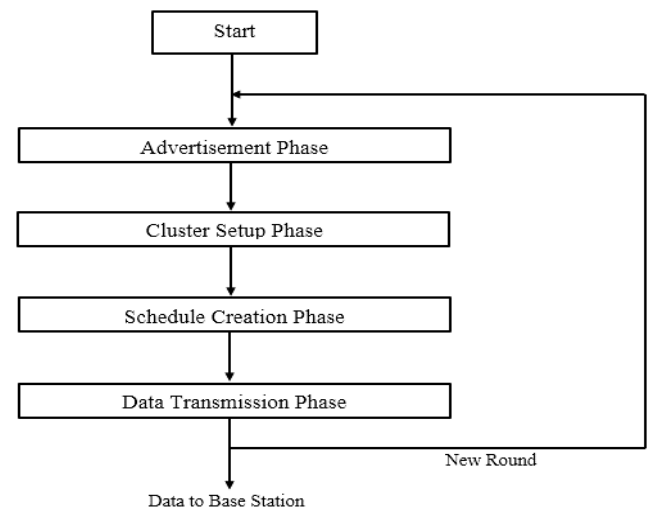

Figure 2. The flowchart of LEACH

\section{MERGED TRANSMITTED DATA LEACH (LEACH-MTD)}

When studying the characteristics of LEACH protocol, we discovered that the cluster head chosen is critical for improving and extending network longevity, data transmission, and energy efficiency. We discovered that in LEACH, the cluster head sends acquired data (from cluster members) to the BS, which consumes energy in every round, and the cluster head is replaced every round, resulting in additional cost for the advertisement of the cluster.

There are protocols [17-18-19] aim to solve the problem caused by replacing the cluster head every round by checking if the remaining energy of $\mathrm{CH}$ still in the specified range, the $\mathrm{CH}$ in the previous round still the $\mathrm{CH}$ in the current round. But these protocols don't resolve periodically transmission from $\mathrm{CH}$ to $\mathrm{BS}$ every round despite This transmission requires the maximum degree of power dissipation. By delaying the transmission phase in the steady-state technique, We don't allow $\mathrm{CH}$ to broadcast the gathered data from its member nodes to the base station every round, but instead keep it for the next selected number of rounds and merge it with the recently acquired data in the next $\mathrm{m}$ number of rounds. As a result, we can cut our energy consumption.

LEACH-MTD' flowchart is exhibited in Figure 3.

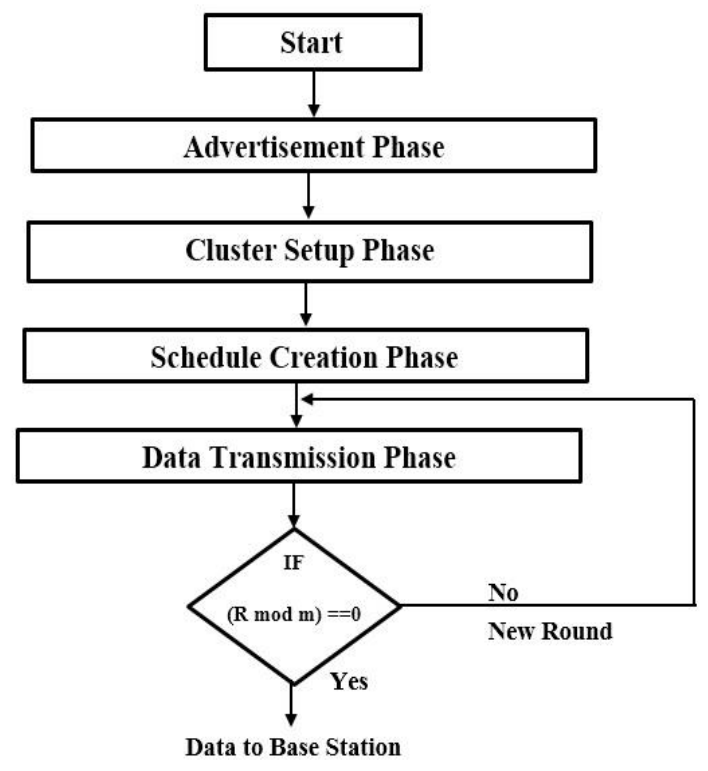

Figure 3. LEACH-MTD flowchart

\section{LEACH VIA SCHEDULE OFF TECHNIQUE WITH MERGING (LEACH-SOTWM)}

Although the first method has greatly improved the lifetime of the network and reduced energy loss, the problem of rising packet size must be avoided. So, we trying to solve this problem by the LEACHSOTWM method.

In the LEACH-SOTWM method, we handle the probability of increasing data packet size if the $\mathrm{CH}$ can't process the collected data from its member nodes by the correct way by making a part of member nodes equal to $(1 / \mathrm{m})$ of rounds where the transmitted data is merged sends the sensing data to its $\mathrm{CH}$ in every round until specified $\mathrm{m}$ rounds, unlike the LEACH-MTD method. We find there is no need for making all member nodes that clustered together to send its sensing data to its $\mathrm{CH}$ because the distance between them is relatively small Consequently, the attached data from nodes is nearby in a similar cluster. By that, we sure that the data packet size still fixed and there is no increase in energy consumption. This method is applicable for application in where no need for periodically report as which measures temperature and humidity and not applicable for applications that need to periodically report as in the Military and in E-Health. So, we select the value of $m$ parameter related to the type of application. 4.

The LEACH-SOTWM' flowchart is declared in Figure 


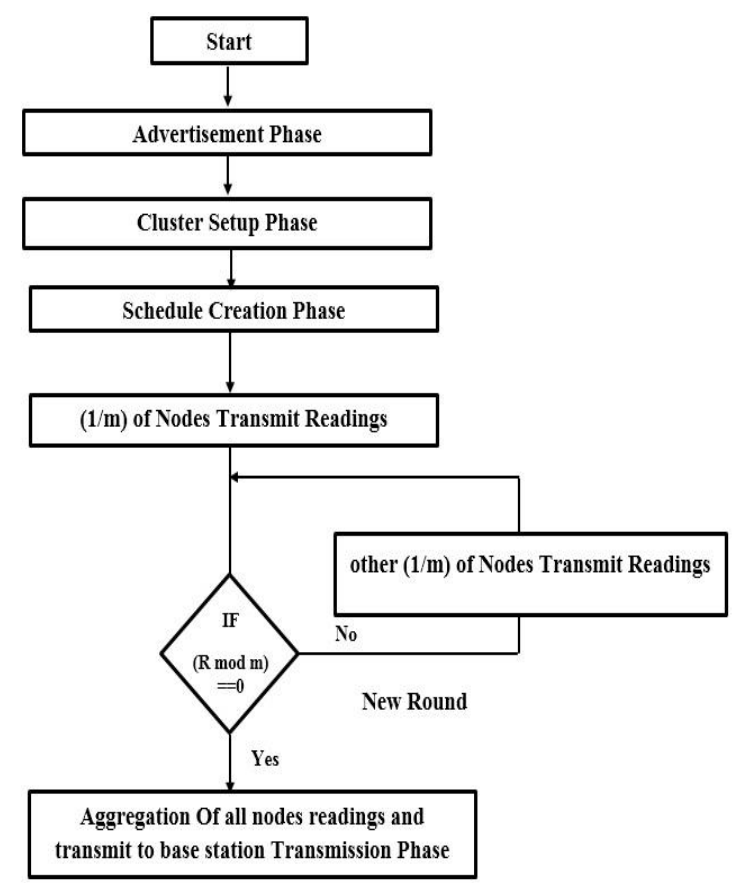

Figure 4. The LEACH-SOTWM flowchart

\section{EXPEREMENTAL METHODS AND RESULTS}

To emphases the greater performance of the suggested method, we did an experiment where the nodes numbers are set as 100. To simulate this algorithm, we used a MATLAB platform.

Yet the network lifetime prolongs still the biggest obstacle in WSN [20] is. As a result, we prefer to prolong the lifetime of the network and present two updates used the same parameters as the LEACH. The parameter values are determined based on earlier research [20-21] that analyses the WSN parameter and chooses the best results for simulations and experiments.

Table 1 below declares the proposed methods of simulation parameters.

Table 1. Factors of Simulation

\begin{tabular}{|l|l|}
\hline Factor & Value \\
\hline Sensor Deployment Area & $100 \times 100 \mathrm{~m}$ \\
\hline Nodes' Number & 100 \\
\hline Packet Size of Data & 4000 bits \\
\hline Control Packet Size & 100 bits \\
\hline $\begin{array}{l}\text { The Sensor's Initial } \\
\text { Energy }\end{array}$ & $0.5 \mathrm{~J}$ \\
\hline $\begin{array}{l}\text { Probability of Each Node } \\
\text { to Become CH }\end{array}$ & 0.1 \\
\hline Maximum round (rmax) & 8000,16000 \\
\hline Merged round number (m) & 2,4 \\
\hline
\end{tabular}

The network lifetime is one of the measuring criteria used to monitor the network life cycle; in this study, we focus on the living nodes in the network. Figures 5 and 6 show the LEACH protocol's living nodes as a function of the number of rounds and our methodologies. When compared to simple LEACH, the proposed approaches extended network lifetime by. It can also be seen in LEACH protocol the number of remaining alive nodes is decreased quickly versus rounds more than our methods because the node death in LEACH is more quickly than in our methods. We notice that increasing $\mathrm{m}$ causes an increase in the lifetime of the network. This increase is in LEACH-MTD and LEACH-SOTWM by $37.75 \%$ and $69.40 \%$ respectively by comparing with their results where $\mathrm{m}=2$.

Figure 7, Figure 8 show the consumed energies number by using LEACH and our methods. It declared that the proposed two update methods decreased energy consumption by comparing it with the LEACH protocol.

A comparison between our proposed methods the LEACH-SOTWM, the LEACH-MTD, the EEE $\mathrm{LEACH}$, and the LEACH protocol from where the First Node Dead, and Last Node Dead is presented in Table 2.

Table 2. Comparison of protocols based for FND, and

\begin{tabular}{|l|l|l|} 
Protocol & $\begin{array}{l}\text { First Node } \\
\text { Dead }\end{array}$ & $\begin{array}{l}\text { Last Node } \\
\text { Dead }\end{array}$ \\
\hline LEACH-SOTWM & 3177 & 7125 \\
\hline LEACH-MTD & 1731 & 6157 \\
\hline EEELEACH & 1374 & 2410 \\
\hline LEACH & 761 & 3422 \\
\hline
\end{tabular}

We were satisfied with comparing the LEACH protocol and our methods only in the figures because our methods achieved results better than the EEE LEACH also.

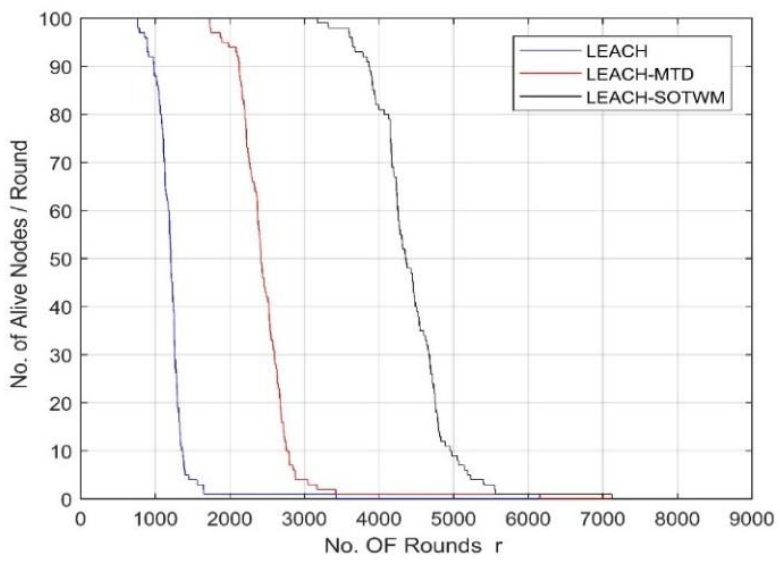

Figure 5. The nodes' number that are alive versus the number of rounds where $m=2$ 


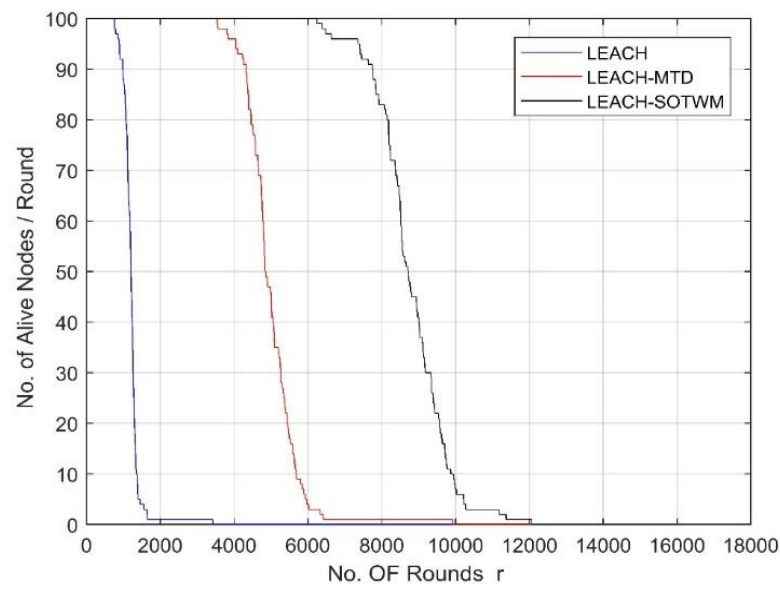

Figure 6. The nodes' number that are alive versus the number of rounds where $m=4$

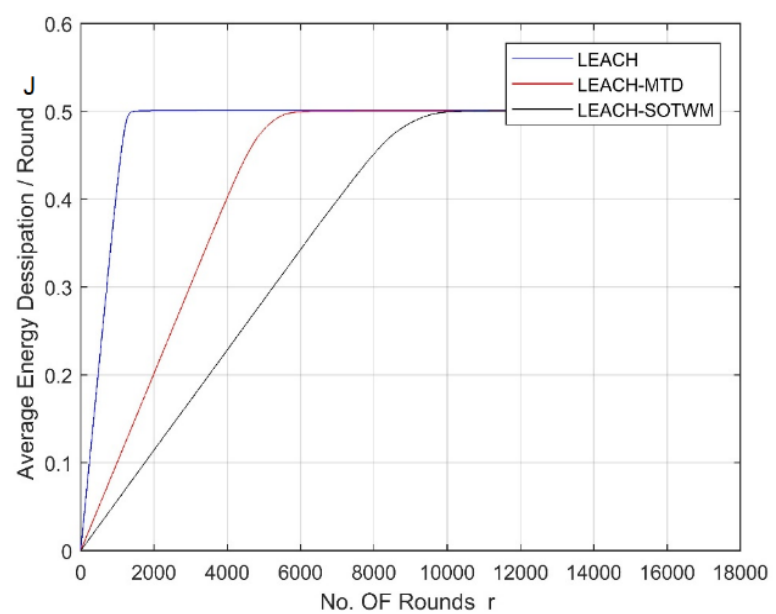

Figure 7. Comparison of the energy dissipation versus No. of rounds where $m=2$

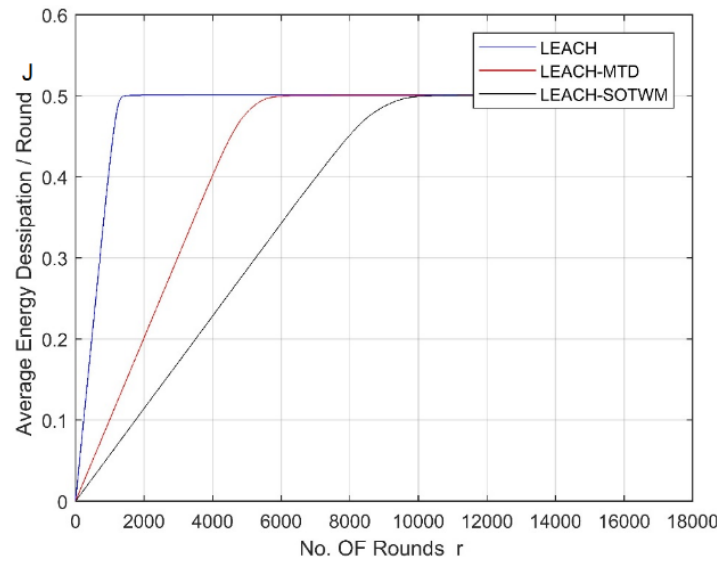

Figure 8. Comparison of the energy dissipation versus No. of rounds where $m=4$

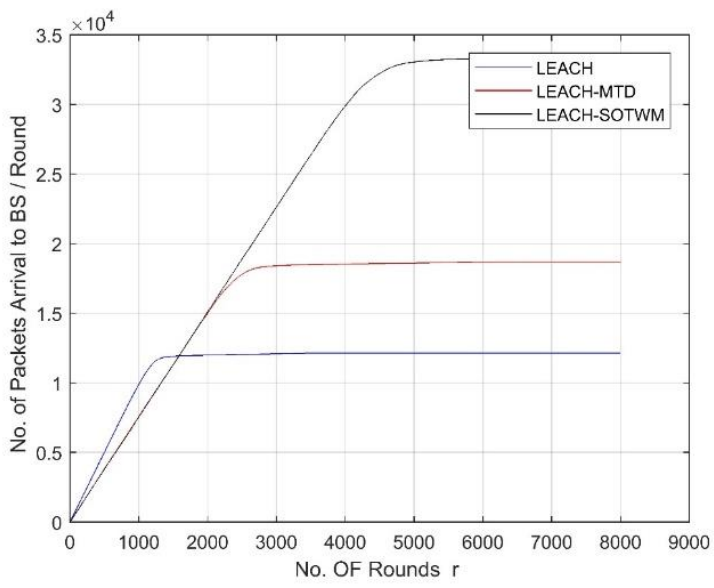

Figure 9. The number of transmitted packets to the BS for 100 nodes where $m=2$

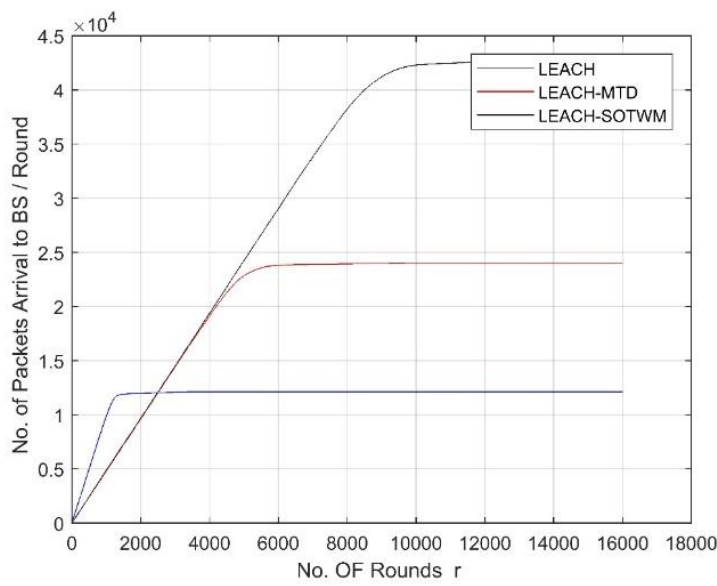

Figure 10. The number of transmitted packets to the BS for 100 nodes where $m=4$

\section{CONCLUSION}

WSNs are used in a wide range of applications. One of the most well-known WSN protocols is the LEACH protocol. In this research, we describe two innovative strategies for reducing energy dissipation and overhead caused by cluster head election and advertisement. The energy in WSNs acts as an important factor in designing, controlling, and operating the sensor networks. Decreasing the consumed energy in WSNs applications is a critical issue for network efficiency in terms of lifetime prolong, cost and operation. The proposed methods increased the lifetime of the network by compared to the LEACH protocol's basic version.

Our work will be used to application strategies with strict coverage requirements in the future, and it will combine our technology with cloud computing.

\section{Credit Authorship Contribution Statement:}

Samar Awad Mohammed: Methodology, Software, and Original draft. Khaled abd Elsalam Aly: Methodology, Conceptualization, Implementation, Results and analysis, Review, and Supervision. Atef Mohammed Ghuniem: Review and Editing. 


\section{Declaration of competing Interest}

The authors declare that they have no known competing financial interests or personal relationships that could have appeared to influence the work reported in this paper.

\section{References}

[1] Gheisari, Mehdi, et al. "A Survey on Clustering Algorithms in Wireless Sensor Networks: Challenges, Research, and Trends". International Computer Symposium (ICS). IEEE, p. 294-299, 2020.

[2] Chintala, Radhika Rani. "Wireless sensor network for m-healthcare monitoring of human being". International Journal, 8.5, 2020.

[3] Engmann, Felicia, et al. "Prolonging the Lifetime of Wireless Sensor Networks: A Review of Current Techniques" Wireless Communications and Mobile Computing, pp. 1-23, 2018.

[4] Rault, Tifenn, AbdelmadjidBouabdallah, and YacineChallal. "Energy efficiency in wireless sensor networks: A top-down survey." Computer networks, vol. 67, pp.104-122, 2014.

[5] Sharma, Naveen, and Anand Nayyar. "A comprehensive review of cluster-based energy efficient routing protocols for wireless sensor networks." International Journal of Application or Innovation in Engineering \& Management (IJAIEM), vol. 3.1, pp.441-453, 2014.

[6] Samara, Ghassan, and Mohammad Aljaidi. "Efficient energy, cost reduction, and QoS based routing protocol for wireless sensor networks." arXiv preprint arXiv:1903.09636, 2019.

[7] ANAND, Santosh; MANOJ, K. C. "A Survey on Clustering Approaches to Strengthen the Performance of Wireless Sensor Network". International Conference on Inventive Research in Computing Applications (ICIRCA). IEEE, p. 814-820, 2020.

[8] M. Quwaider and S. Biswas. "Modeling energy harvesting sensors using accelerometer in body sensor networks". in Proc. 8th International Conf. Body Area Networks, pp. 148-152, 2013.

[9] Sharma, Nishi, and Vandna Verma. "Energy-efficient LEACH protocol for wireless sensor network". International Journal of Information and Network Security, 2.4: 333, 2013.

[10] Prasad, A. Y., and R. Balakrishna. "Implementation of optimal solution for network lifetime and energy consumption metrics using improved energy efficient LEACH protocol in MANET." Telkomnika, 17.4, pp. 1758-1766, 2019.

[11] Nandi, Arnab. "Centered sink leach protocol for enhanced performance of wireless sensor network". In: 2019 International Conference on Automation, Computational and Technology Management (ICACTM). IEEE. pp. 436-440, 2019.

[12] Sharma, Meenakshi, and Anil Kumar Shaw. "Transmission time and throughput analysis of EEE LEACH, LEACH and direct transmission protocol: a simulation-based approach." Advanced Computing, 3.6: 75, 2012.

[13] Sharma, Rohini, Narendra Mishra, and Sumit Srivastava. "A proposed energy efficient distancebased cluster head (DBCH) Algorithm: An Improvement over LEACH." Procedia Computer Science, 57, 807-814, 2015.

[14] Heinzelman, Wendi Rabiner, AnanthaChandrakasan, and Hari Balakrishnan. "Energy-efficient communication protocol for wireless microsensor networks." Proceedings of the 33rd annual Hawaii international conference on system sciences. IEEE, vol.2, p. 10 pp, 2000.

[15] Fu, Chunyao, et al. "An energy balanced algorithm of LEACH protocol in WSN." International Journal of Computer Science Issues (IJCSI), vol. 10, no. 1, p. 354, 2013.

[16] Kemal Akkaya and Mohamed Younis. "A survey on routing protocols for wireless sensor networks". Ad hoc networks,3.3,325-349, 2005.

[17] Mr. Santosh N. Shelke, Mr. Sandip R. Shinde. "Energy saving techniques in wireless sensor networks". International Journal of Scientific \& Engineering Research, Vol. 4, Issue. 4, 2013.

[18] Ponnusamy, Vasaki. "Energy analysis in Wireless Sensor Network: a comparison". International Journal of Computer Networks and Communications Security. International Journal of Computer Networks and Communications Security, vol.2, pp.328-338, 2014.

[19] Liang, , Haibo, et al. "Research on routing optimization of WSNs based on improved LEACH protocol". EURASIP Journal on Wireless Communications and Networking, vol.1, pp. 1-12, 2019.

[20] NASR, Seham; QUWAIDER, Muhannad. "LEACH Protocol Enhancement for Increasing WSN Lifetime". International Conference on Information and Communication Systems (ICICS). IEEE, p. 102-107, 2020. 